\title{
Hierarchical and Recursive State Machines with Context-Dependent Properties ${ }^{\star}$
}

\author{
Salvatore La Torre, Margherita Napoli, Mimmo Parente, and \\ Gennaro Parlato \\ Dipartimento di Informatica e Applicazioni \\ Università degli Studi di Salerno
}

\begin{abstract}
Hierarchical and recursive state machines are suitable abstract models for many software systems. In this paper we extend a model recently introduced in literature, by allowing atomic propositions to label all the kinds of vertices and not only basic nodes. We call the obtained models context-dependent hierarchical/recursive state machines. We study on such models cycle detection, reachability and LTL modelchecking. Despite of a more succinct representation, we prove that LTL model-checking can be done in time linear in the size of the model and exponential in the size of the formula, as for standard LTL model-checking. Reachability and cycle detection become NP-complete, and if we place some restrictions on the representation of the target states, we can decide them in time linear in the size of the formula and the size of the model.
\end{abstract}

Keywords: Model Checking, Automata, Temporal Logic.

\section{Introduction}

Due to their complexity, the verification of the correctness of many modern digital systems is infeasible without suitable automated techniques. Formal verification has been very successful and recent results have led to the implementation of powerful design tools (see CK96). In this area one of the most successful techniques has been model checking [CE81]: a high-level specification is expressed by a formula of a logic and this is checked for fulfillment on an abstract model (state machine) of the system. Though model checking is linear in the size of the model, it is computationally hard since the model generally grows exponentially with the number of variables used to describe a state of the system (state-space explosion). As a consequence, an important part of the research on model checking has been concerned with handling this problem.

Complex systems are usually composed of relatively simple modules in a hierarchical manner. Hierarchical structures are also typical of object-oriented

\footnotetext{
* This research was partially supported by the MIUR in the framework of the project "Metodi Formali per la Sicurezza e il Tempo" (MEFISTO) and MIUR grant 60\% 2002.
} 
paradigms [BJR97 $\mathrm{RBP}^{+}$91 SGW94]. We consider systems modeled as hierarchical finite state machines, that is, finite state machines where a vertex can either expand to another hierarchical state machine or be a basic vertex (in the former case we call the vertex a supernode, in the latter simply a node).

The model we consider in this paper generalizes instead the model studied in AY01. There the authors consider the model checking on Hierarchical State Machines (HSM) where only the nodes are labeled with atomic propositions $(A P)$. We relax this constraint and thus we allow to associate atomic propositions also with vertices that expand to a machine. Expanding a supernode $v$ to a machine $M$, all vertices of $M$ inherit the atomic propositions of $v$ (context), so that different vertices expanding to $M$ can place $M$ into different contexts. For this reason, we call such a model a hierarchical state machine with context-dependent properties (in the following denoted by Context-dependent Hierarchical State Machine). The semantics of a CHSM is given by the corresponding natural flat model which is a Kripke structure.

By allowing this more general labeling, for a given system it is possible to obtain very succinct abstract models. In the following example, we show that the gain of succinctness can be exponential compared to the models used in AY01. Consider a digital clock with hours, minutes, and seconds. We can construct a hierarchical finite state machine $\mathcal{M}$ composed of three machines $M_{1}, M_{2}$, and $M_{3}$ such that the supernodes of $M_{3}$ expands to $M_{2}$ and the supernodes of $M_{2}$ expands to $M_{1}$. Machine $M_{1}$ is a chain of nodes. Machines $M_{2}$ and $M_{3}$ are chains of supernodes except for the initial and the output vertices that are nodes. In $M_{3}$ each supernode corresponds to a hour and they are linked accordingly to increasing time. Analogously, $M_{2}$ models minutes and $M_{1}$ seconds. A flat model for the digital clock has at least $24 \cdot 60 \cdot 60=86,400$ vertices, while the above hierarchical model has only $24+60+60+6=150$ vertices $(6$ are simply initial and output nodes). Assume that we are interested in checking properties that refer to a precise time expressed in hours, minutes and seconds. Clearly, it is not sufficient to label only the nodes (we would be able to capture only that an event happens at a certain second, but we would have no clue of the actual hour and minute). In the model defined in [AY01, at least 86,400 nodes are needed, that is, there would be no gain with respect to a minimal flat model. In our model, we are able to label each supernode in $M_{3}$ with atomic propositions encoding the corresponding hour. Analogously we can use atomic propositions to encode minutes and seconds on $M_{2}$ and $M_{1}$, respectively. This way, each state of the corresponding flat model is labeled with the encoding of a hour, a minute and a second in a day and vertices are linked by increasing time.

A simple way of analyzing hierarchical systems is first to flatten them into equivalent non-hierarchical systems and then apply existing verification techniques on finite state systems. The drawback of such an approach is that the size of the flat system can be exponential in the hierarchical depth. In many recent papers, it has been shown that it is possible to reduce the complexity growth caused by handling large systems, by performing verification in a hierarchical manner AGM00 AG00 BLA ${ }^{+}$99 AY01. We follow this approach and study on 
CHSMs standard decision problems which are related to system verification, such as reachability, cycle detection, and model checking. In this paper, we also consider Context-dependent Recursive State Machines (CRSM) which generalize CHSMs by allowing recursive expansions and we study on them the verification-related problems listed above. Recursive generalizations of the hierarchical model presented in AY01 are studied in AEY01BGR01. Recursive machines can be used to model the control flow of programs with recursive calls and thus are suitable for abstracting the behavior of reactive software systems. Results. Given a transition system, a state $s$ and a set of target states $T$, (usually expressed by a propositional boolean formula), the reachability problem is the problem of determining whether a state of $T$ can be reached from $s$ on a run of the system. In practice, this problem is relevant in the verification of systems, for example it is related to the verification of safety requirements: we want to check whether all the reachable states of the system belong to a given "safe" region (invariant checking problem).

We prove that reachability on CRSMs is NP-complete, and NP-hardness still holds if we restrict to CHSMs. We then give an algorithm to decide reachability on CRSMs that runs in time linear in the size of the model and exponential in the size of the formula. Finally, given a CHSM $\mathcal{M}$, we show effective sufficient conditions for solving reachability in time linear in both the size of the formula and the size of the model. Let us remark that these conditions are satisfied when we consider an instance of the reachability problem where the model is given by a Hierarchical State Machine (HSM) as defined in AY01.

The cycle detection problem is the problem of verifying whether a given state can be reached repeatedly. Cycle detection is the basic problem for the verification of liveness properties: "some good thing will eventually happen".

We also consider the model checking of LTL formulas on CRSMs. Given a set of atomic propositions $A P$, a linear temporal logic (LTL) formula is built up in the usual way from atomic propositions, the boolean connectives, the temporal operators next and until. An LTL formula is interpreted over an infinite sequence over $2^{A P}$. A CRSM satisfies a formula $\varphi$ if every run in the corresponding flat model satisfies $\varphi$. Given an LTL formula $\varphi$ and a $\mathbf{C R S M} \mathcal{M}$, the model checking problem for $\mathcal{M}$ and $\varphi$ is the problem to determine whether $\mathcal{M}$ satisfies $\varphi$. We give a decision algorithm that runs in $O\left(|\mathcal{M}| \cdot 8^{|\varphi|}\right)$ time for CHSMs and an algorithm in $O\left(|\mathcal{M}| \cdot 16^{|\varphi|}\right)$ time for CRSMs. Our algorithms do not need to flatten the system and mainly consist of reducing the model checking problem to the emptiness problem of recursive Büchi automata AEY01.

The rest of the paper is organized as follows. In the next section definitions and notation are given. The NP-completeness of the cycle detection and of the reachability problems is shown in section 3 (actually the proofs for the cycle detection problems are omitted in this version, due to the lack of space). In section 4 we give the linear time algorithms for CHSMs and CRSMs. In Section 5 we discuss the model checking of LTL formulas. We conclude with few remarks in Section 6. 


\section{Context-Dependent State Machines}

In this section we introduce the definitions and the notation we will use in the rest of the paper. We consider Kripke structures, that is, state-transition graphs where each state is labeled by a subset of a finite set of atomic propositions $(A P)$. A Context-dependent Recursive State Machine (CRSM) over $A P$ is a tuple $\mathcal{M}=\left(M_{1}, \ldots, M_{k}\right)$ of Kripke structures with:

- a set of vertices $N$, split into disjoint sets $N_{1}, \ldots, N_{k}$; a set $I N=$ $\left\{i n_{1}, \ldots, i n_{k}\right\}$ of initial vertices, where $i n_{i} \in N_{i}$, and a set of output vertices $O U T$ split into $O U T_{1}, \ldots, O U T_{k}$, with $O U T_{i} \subseteq N_{i}$;

- a mapping expand $: N \longrightarrow\{0,1, \ldots, k\}$ such that $\operatorname{expand}(u)=0$, for each $u \in I N \cup O U T$. We define the closure of expand, expand ${ }^{+}: N \longrightarrow 2^{\{0,1, \ldots, k\}}$, as: $h \in \operatorname{expand}^{+}(u)$ if either $h=\operatorname{expand}(u)$ or $u^{\prime} \in N_{\text {expand(u) }}$ exists such that $h \in \operatorname{expand}^{+}\left(u^{\prime}\right)$.

- the sets of edges $E_{i}$, for $1 \leq i \leq k$, such that each edge in $E_{i}$ is either a pair $(u, v)$, with $u, v \in N_{i}$ and expand $(u)=0$, or a triple $((u, z), v)$ with $z \in O U T_{\text {expand }(u)}$, and $u, v \in N_{i}$;

- a mapping true : $N \longrightarrow 2^{A P}$, such that $\operatorname{true}(u) \cap \operatorname{true}(v)=\emptyset$, for $v \in$ $N_{h}, u \notin N_{h}$ and $h \in \operatorname{expand}^{+}(u)$.

Informally, a CRSM is a collection of graphs which can call each other recursively. Each graph has an initial vertex and some output vertices. The mapping expand gives the recursive-call structure. If $\operatorname{expand}(u)=j>0$, then the vertex $u$ expands to the graph $M_{j}$ and $u$ is called a supernode; when expand $(u)=0$ the vertex $u$ is called a node. The mapping true labels each vertex with a set of atomic propositions holding at that vertex. The starting node of a CRSM $\mathcal{M}=\left(M_{1}, \ldots, M_{k}\right)$ is the initial node $i n_{k}$ of $M_{k}$.

The Semantics of CRSMs. Every CRSM $\mathcal{M}$ corresponds to a flat model $\mathcal{M}^{F}$ which is a directed graph with (possibly infinite) vertices (states) labeled with atomic propositions. Informally speaking, the flat machine $\mathcal{M}^{F}$ is obtained starting from $M_{k}$ and iteratively replacing every supernode $u$ in it with the graph $M_{\text {expand }(u)}$. The flat machine $\mathcal{M}^{F}$ is defined as follows. A state of $\mathcal{M}^{F}$ is a tuple $X=\left[u_{1}, \ldots, u_{m}\right]$ where $u_{1} \in N_{k}, u_{j+1} \in N_{\operatorname{expand}\left(u_{j}\right)}$ for $j=1, \ldots, m-1$, and $\operatorname{expand}\left(u_{m}\right)=0$. State $X$ is labeled by a set of atomic proposition $\operatorname{true}(X)$, consisting of the union of $\operatorname{true}\left(u_{j}\right)$, for $j=1, \ldots, m$. State $\left[i n_{k}\right]$ is the initial state of $\mathcal{M}^{F}$. The set of transitions $E$ is defined as follows. Let $X=\left[u_{1}, \ldots, u_{m}\right]$ be a state with $u_{m} \in N_{h}$ and $u_{m-1} \in N_{j}$. Then, $\left(X, X^{\prime}\right) \in E$ provided that one of the following cases holds:

1. $\left(u_{m}, u^{\prime}\right) \in E_{h}, u^{\prime} \in N_{h}$, and if $\operatorname{expand}\left(u^{\prime}\right)=0$ then $X^{\prime}=\left[u_{1}, \ldots, u_{m-1}, u^{\prime}\right]$, otherwise $X^{\prime}=\left[u_{1}, \ldots, u_{m-1}, u^{\prime}, i n_{l}\right]$ for $l=\operatorname{expand}\left(u^{\prime}\right)$.

2. $u_{m} \in O U T_{h},\left(\left(u_{m-1}, u_{m}\right), u^{\prime}\right) \in E_{j}, u^{\prime} \in N_{j}$, and if $\operatorname{expand}\left(u^{\prime}\right)=0$ then $X^{\prime}=\left[u_{1}, \ldots, u_{m-2}, u^{\prime}\right]$, otherwise $X^{\prime}=\left[u_{1}, \ldots, u_{m-2}, u^{\prime}, i n_{l}\right]$ for $l=\operatorname{expand}\left(u^{\prime}\right)$.

Let $\left[u_{1}, \ldots, u_{n}\right]$ be a state of $\mathcal{M}^{F}$, a prefix of $\left[u_{1}, \ldots, u_{n}\right]$ is $u_{1}, \ldots, u_{i}$ for $i \leq n$. 
A Context-dependent Hierarchic State Machine (CHSM) is a CRSM such that $\operatorname{expand}(u)<i$, for every $u \in N_{i}$. A CHSM is a collection of graphs which are organized to form a hierarchy and expand gives the hierarchical structure. The graph $M_{k}$ is clearly the top-level graph of the hierarchy, i.e., no vertices expand to it and, as for CRSMs, its initial node $i n_{k}$ is the starting node of the CHSM.

\section{Reachability and Cycle Detection Problems: Computational Complexity}

In this section we discuss the computational complexity of the reachability and cycle detection problems for CRSMs and CHSMs. Given a CRSM $\mathcal{M}=$ $\left(M_{1}, \ldots, M_{k}\right)$ and a propositional boolean formula $\varphi$, the reachability problem is the problem of deciding if a path in $\mathcal{M}^{F}$ exists from $\left[i n_{k}\right]$ to a state $X$ on which $\varphi$ is satisfied. Analogously, the cycle detection problem is the problem of deciding if a cycle in $\mathcal{M}^{F}$ exists containing a reachable state $X$ on which $\varphi$ is satisfied.

We prove that for CRSMs and CHSMs these decision problems are NPcomplete by showing NP-hardness for CHSMs and giving nondeterministic polynomial-time algorithms for CRSMs.

Lemma 1. Reachability and cycle detection for $\mathbf{C H S M} s$ are NP-hard.

Proof We give a reduction in linear time with respect to the size of the formula from the satisfiability problem SAT. Given a boolean formula $\varphi$ over the variables $x_{1}, \ldots, x_{m}$, we construct a CHSM $\mathcal{M}=\left(M_{1}, M_{2}, \ldots, M_{m}\right)$ over $A P=\left\{P_{1}, P_{2}, \ldots, P_{m}\right\}$, as follows. Each graph $M_{i}$ has four vertices $i n_{i}, p_{i}$, not $_{i}$, out $_{i}$ forming a chain. Each vertex $p_{i}$ is labeled by $\left\{P_{i}\right\}$ whereas the vertices $\operatorname{notp}_{i}, i n_{i}$ and $o u t_{i}$ are labeled by the empty set. Since an atomic proposition $P_{i}$ does not label vertices in graphs other than $M_{i}$, this labeling implicitly corresponds to assigning $\neg P_{i}$ to $\operatorname{not} p_{i}$. Vertices $p_{i}$ and $\operatorname{not} p_{i}$, for $i>1$, are supernodes which expand into $M_{i-1}$, and $p_{1}$ and not $p_{1}$ are instead nodes.

Thus there are $2^{m}$ states of $\mathcal{M}^{F}$ of type $\left[u_{1}, \ldots, u_{m}\right]$ such that $u_{m-i+1} \in$ $\left\{p_{i}, \operatorname{not}_{i}\right\}$ for $i=1, \ldots, m$, and it is easy to verify that all these states are reachable from $\left[i n_{m}\right]$. Clearly, given a truth assignment $\nu$ of $x_{1}, \ldots, x_{m}$, a state $X$ of $\mathcal{M}^{F}$ exists such that $\nu$ assigns TRue to $x_{i}$ if and only if $p_{i}$ occurs in $X$ and, in turns, if and only if $P_{i} \in \operatorname{true}(X)$. Thus a reachable state $X$ of $\mathcal{M}^{F}$ exists whose labeling corresponds to a truth assignment fulfilling $\varphi$ if and only if $\varphi$ is satisfiable.

By definition of the cycle detection problem, checking for the existence of a cycle containing a state on which $\varphi$ is satisfied requires to check for reachability first. Thus, NP-hardness is inherited from reachability.

To prove membership to NP of the reachability on CRSMs, we need to consider a notion of connectivity of vertices in a CRSM. We say that a vertex $u \in N$ is connected if a reachable state $\left[u_{1}, \ldots, u_{m}\right]$ of $\mathcal{M}^{F}$ exists, where $u=u_{i}$ for some $i=1, \ldots, m$. Observe that the starting node $i n_{k}$ is clearly connected 
and a vertex $u \in N_{j}$ is connected if and only if $i n_{j}$ is connected and a path $\pi$ in $M_{j}$ from $i n_{j}$ to $u$ exists, such that if $\pi$ goes through an edge $\left((v, z), v^{\prime}\right) \in E_{j}$ then $z$ is a connected vertex (recall that $z \in \mathrm{OUT}_{\text {expand }(v)}$ ). From this the following proposition holds.

Proposition 1. A state $\left[u_{1}, \ldots, u_{m}\right]$ of $\mathcal{M}^{F}$ is reachable if and only if all the vertices $u_{i}$, for $i=1, \ldots, m$, are connected.

The above observation suggests also an algorithm to determine in linear time the connected vertices. We omit the proof of this result which is given by a rather simple modification of a depth-first search on a graph (see also [AEY01]).

Proposition 2. Given a $\mathbf{C R S M} \mathcal{M}$, the set of connected vertices of $\mathcal{M}$ can be determined in $O(|\mathcal{M}|)$.

To prove membership to NP of the reachability on CRSMs, we need to prove the following technical lemma. Notice that this lemma is not needed for CHSMs, where the number of supernodes that compose a state of $\mathcal{M}^{F}$ is bounded from above by the number of component graphs.

Lemma 2. Given a CRSM $\mathcal{M}$, for each state $X=\left[u_{1}, \ldots, u_{m}\right]$ such that $m>n^{2}+1$, where $n$ is the number of supernodes of $\mathcal{M}$, a state $X^{\prime}=\left[u_{1}^{\prime}, \ldots, u_{m^{\prime}}^{\prime}\right]$ exists such that $m^{\prime}<m$ and true $(X)=\operatorname{true}\left(X^{\prime}\right)$. Moreover, if $X$ is reachable then also $X^{\prime}$ is reachable.

Proof Consider a sequence $v_{1}, \ldots, v_{h} \in N$. We say that a sub-sequence $v_{i} \ldots v_{j}$, $1 \leq i<j$, is a cycle if $v_{i}=v_{j}$. Moreover, we say that a cycle $v_{i} \ldots v_{j}$, is erasable if $\left\{v_{i+1}, \ldots, v_{j}\right\} \subseteq\left\{v_{1}, \ldots, v_{i}\right\}$. It is easy to verify that for a sequence $u_{1} \ldots u_{m}$ such that $X=\left[u_{1}, \ldots, u_{m}\right]$ is a state of $\mathcal{M}^{F}$ and $u_{i} \ldots u_{j}$ is a cycle, we have that $X^{\prime}=\left[u_{1}, \ldots, u_{i}, u_{j+1}, \ldots, u_{m}\right]$ is a state of $\mathcal{M}^{F}$ and if $u_{i} \ldots u_{j}$ is also erasable then $\operatorname{true}(X)=\operatorname{true}\left(X^{\prime}\right)$. Moreover, by Proposition 1 if $X$ is reachable then also $X^{\prime}$ is reachable.

To conclude the proof we only need to show that for each state $X=$ $\left[u_{1}, \ldots, u_{m}\right]$ such that $m>n^{2}+1$, where $n$ is the number of supernodes in $\mathcal{M}, u_{1} \ldots u_{m}$ contains an erasable cycle. Notice that $m>n^{2}+1$ implies that a supernode $u$ exists, occuring at least $(n+1)$ times in $u_{1} \ldots u_{m}$. Suppose $u_{1} \ldots u_{m}=\alpha_{0} u \alpha_{1} u \ldots \alpha_{n} u \beta$, where each $\alpha_{i}$ does not contain occurrences of $u$. A cycle $u \alpha_{i} u$ is not erasable only if it contains a supernode that is not in $\alpha_{0} u \ldots \alpha_{i-1} u$. By a simple count, if $\alpha_{0} u \ldots \alpha_{n-1}$ does not contain erasable cycles, then all supernodes occur in it. Thus, $u \alpha_{n} u$ is erasable.

Now, we can prove membership to NP of the reachability and the cycle detection problems on CRSMs.

Lemma 3. Reachability and cycle detection for $\mathbf{C R S M} s$ are decidable in nondeterministic polynomial-time.

Proof Consider the instance of the reachability problem given by a CRSM $\mathcal{M}$ and a propositional boolean formula $\varphi$. By Proposition 2 we can determine in 
$O(|\mathcal{M}|)$ time the set of the connected vertices, and then, given a state $X$ of $\mathcal{M}^{F}$, by Proposition 1 we can check if $X$ is reachable in $O(|\mathcal{M}|+|X|)$ time. Verifying the fulfillment of $\varphi$ on $X$ takes $O(|\varphi|+|X|)$ time. Moreover, by Lemma2 we need only to consider states $X=\left[u_{1}, \ldots, u_{m}\right]$ for $m \leq n^{2}+1$, where $n$ is the number of supernodes of $\mathcal{M}$. Thus, we can conclude that the reachability problem on CHSMs is in NP.

By Lemmas 1 and 3 we have the following theorem.

Theorem 1. Reachability and cycle detection for $\mathbf{C R S M}$ (CHSMs) are NPcomplete.

\section{Efficient Solutions to Reachability and Cycle Detection Problems}

In this section, we give a linear time algorithm that solves reachability and cycle detection problems for CHSMs which are related to target sets by a particular condition (specified later). As a corollary we get three consequences: first the results regarding reachability and cycle detection for the model considered in [AY01] are obtained as particular cases, second we characterize a class of formulas guaranteeing that the algorithm works correctly and finally we show that the algorithm works also for DNF formulas, thus obtaining a general solution for any formula with a tight worst case running time of $O\left(|\mathcal{M}| \cdot 2^{|\varphi|}\right)$.

Finally, we give a linear time reduction from the reachability problem on CRSMs for DNF formulas to the corresponding problem on CHSMs, thus the above general solution still holds for CRSMs.

Consider now CHSMs. Clearly a propositional formula $\varphi$ can be evaluated in a state $X$ of $\mathcal{M}^{F}$ by instantiating to TRUE the variables corresponding to the atomic propositions in $\operatorname{true}(X)$ and to FALSE all the others. Now we wish to evaluate $\varphi$ without constructing the graph $\mathcal{M}^{F}$, to this aim we use a greedy approach in a top-down fashion on the hierarchy: at each supernode we instantiate as many variables as possible. By traversing the hierarchy in a top-down fashion, once a node is reached, $\varphi$ can only partially evaluated. On a supernode $u$ of a CHSM all the variables instantiated to TRUE correspond to the atomic propositions in true $(u)$. Determining the variables to instantiate to FALSE is not so immediate. We define $A P(h)$ as the union of the sets labeling either the vertices in $N_{h}$ or those having an ancestor in $N_{h}$, that is, $A P(h)=\bigcup_{v \in N_{h}}(\operatorname{true}(v) \cup A P(\operatorname{expand}(v)))$ where $A P(0)=\emptyset$. Moreover, for $u \in N_{h}$, we define the set $f a l s e(u)$ as $A P(h) \backslash(\operatorname{true}(u) \cup A P(\operatorname{expand}(u)))$. This set contains the atomic propositions that can be instantiated to FALSE at $u$, since a proposition $p \in \operatorname{false}(u)$ if and only if $p \notin \operatorname{true}(X)$, for every state $X$ of $\mathcal{M}^{F}$ having the supernode $u$ as a component. It is easy to see that the sets false $(u), u \in N$, can be preprocessed in time $O(|\mathcal{M}|)$, by visiting $\mathcal{M}$ in a bottom-up way.

For a propositional boolean formula $\varphi$ we denote by $\operatorname{Eval}(\varphi, u)$ the formula obtained by instantiating $\varphi$ with $\operatorname{true}(u)$ and false $(u)$. We generalize this notation to sequences of vertices defining $\operatorname{Eval}\left(\varphi, u_{1}, \cdots, u_{i}\right)$ as 


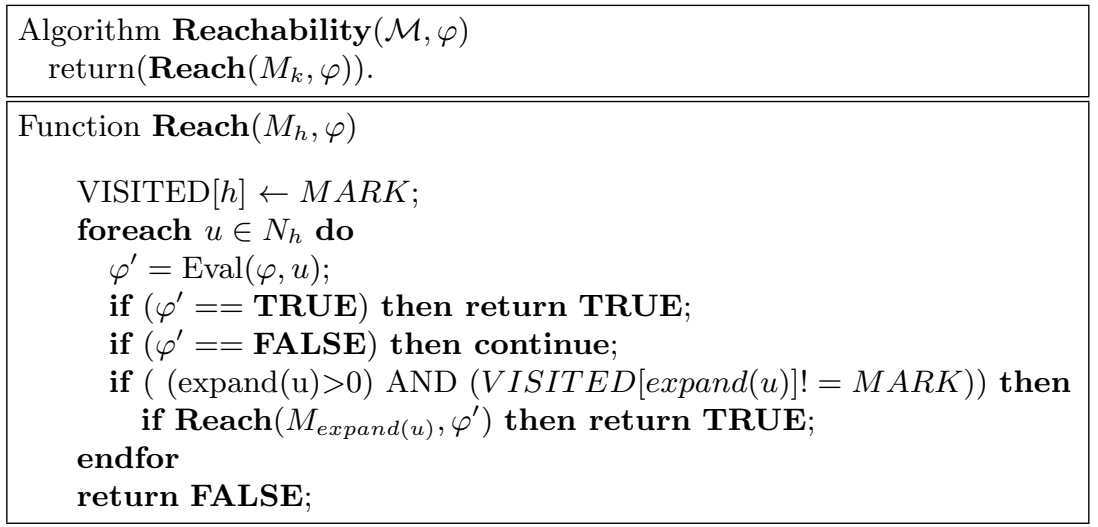

Fig. 1. Algorithm Reachability.

$\operatorname{Eval}\left(\operatorname{Eval}\left(\varphi, u_{1}\right), u_{2}, \cdots, u_{i}\right)$. Finally, we will denote by $A P(\varphi)$ the set of atomic propositions corresponding to $\varphi$ variables.

We consider a condition relating a CHSM $\mathcal{M}$ and a target set specified by a formula $\varphi$ asserting that "when two supernodes expand to the same graph, then any partial evaluation of $\varphi$ ending on them coincides". Formally, the condition is as follows:

Condition 1 Let $x_{1}, \cdots, x_{i}$ and $y_{1}, \cdots, y_{j}$ be two prefixes of $\mathcal{M}^{F}$ states such that expand $\left(x_{i}\right)=\operatorname{expand}\left(y_{j}\right)$. If neither $\operatorname{Eval}\left(\varphi, x_{1}, \cdots, x_{i}\right)$ nor $\operatorname{Eval}\left(\varphi, y_{1}, \cdots, y_{j}\right)$ is one of the constants $\{T R U E, F A L S E\}$, then $\operatorname{Eval}\left(\varphi, x_{1}, \cdots, x_{i}\right)=\operatorname{Eval}\left(\varphi, y_{1}, \cdots, y_{j}\right)$.

When reachability and cycle detection become tractable.

Theorem 2. The reachability and cycle detection problems on a CHSM $\mathcal{M}$ and a formula $\varphi$ satisfying Condition 1 are decidable in time $O(|\mathcal{M}| \cdot|\varphi|)$.

Proof Consider a CHSM $\mathcal{M}=\left(M_{1}, \ldots, M_{k}\right)$ and without loss of generality assume that all the vertices of $\mathcal{M}$ are connected (see Proposition2). Algorithm $\operatorname{Reachability}(\mathcal{M}, \varphi)$ (Figure 1), returns TRUE if and only if $\varphi$ is evaluated to TRUE on a reachable state of $\mathcal{M}^{F}$. The function Reach uses a global array VISITED (initially unmarked in all the positions) to mark the visited graphs $M_{h}$. For each node $u$ of $M_{h}, \varphi$ is evaluated on it according to $\operatorname{true}(u)$ and false $(u)$, call $\varphi^{\prime}$ the returned formula. If $\varphi^{\prime}$ evaluates TRUE on $u$, then Reach stops returning TRUE. (and the main algorithm stops too returning TRUE). If $\varphi^{\prime}$ evaluates FALSE, another vertex of $M_{h}$ which has not yet been explored is processed. In case $u$ is a supernode and $M_{\text {expand }(u)}$ has never been visited, then the function is called on the graph $M_{\text {expand }(u)}$ and $\varphi^{\prime}$. Now note that Condition 1 assures that it is not necessary to visit a graph $M_{h}$ more than once, thus the overall complexity of the algorithm is linear in $|\mathcal{M}|$ and $|\varphi|$ and clearly returns TRUE if and only if a node $X$ in $\mathcal{M}^{F}$ exists on which $\varphi$ is TRUE. 
It is easy to see that given any formula $\varphi$ and a Hierarchical State Machine (HSM) introduced in AY01 (where only nodes are labeled with the mapping true, see the introduction), Condition 1 always holds, thus the linear time solutions for the reachability and cycle detection problems for HSM given in that paper are here obtained as particular cases.

Now we present a characterization of formulas for which Theorem 2 holds. A propositional boolean formula $\varphi$ is said to be in $\mathcal{M}$-normal form if $\varphi=$ $\varphi_{1} \wedge \ldots \wedge \varphi_{m}$ and for every $\varphi_{i}$ and for every vertex $u$ of $\mathcal{M}$ it holds that either $A P\left(\varphi_{i}\right) \cap(\operatorname{true}(u) \cup$ false $(u))=\emptyset$ or $A P\left(\varphi_{i}\right) \cap(\operatorname{true}(u) \cup$ false $(u))=A P\left(\varphi_{i}\right)$. It is easy to see that also in this case Condition 1 holds.

Theorem 2 can be generalized for a finite disjunction of formulas satisfying Condition 1. Since a conjunction of literals is in $\mathcal{M}$-normal form, for all possible $\mathcal{M}$, then this generalization can be applied to DNF formulas. Thus, as any formula $\varphi$ can always be transformed in a DNF formula, we have an algorithm for reachability and cycle detection problems whose worst case running time is $O(|\mathcal{M}| \cdot \operatorname{DNF}(\varphi))$, where $\operatorname{DNF}(\varphi)$ is the cost of the transformation of $\varphi$ in Disjunctive Normal Form. All this yields a tight upper bound of $O\left(|\mathcal{M}| \cdot 2^{|\varphi|}\right)$.

Reachability and cycle detection are also tractable on CRSMs if we restrict to formulas in disjunctive normal form as shown in the following theorem.

Theorem 3. Reachability and cycle detection problems for a CRSM $\mathcal{M}$ and a formula $\varphi$ in DNF are decidable in time $O(|\mathcal{M}| \cdot|\varphi|)$.

Proof Consider a $\operatorname{CRSM} \mathcal{M}$ and a DNF formula $\varphi=\psi_{1} \vee \ldots \vee \psi_{m}$ where each $\psi_{i}$ is a conjunction of literals. Our algorithm consists of reducing in $O(|\varphi| \cdot|\mathcal{M}|)$ time the reachability problem for $\mathcal{M}$ and $\psi_{i}$ to the reachability problem for a CHSM $\overline{\mathcal{M}}$ and $\psi_{i}$, where size of $\overline{\mathcal{M}}$ is $O(|\mathcal{M}|)$. Then the result follows from Theorem 2 .

Consider a disjunct clause $\psi$ of $\varphi$. We simplify $\mathcal{M}$ using the following two steps.

1. for each graph $M_{i}$, delete all the existing edges and insert an edge from $i n_{i}$ to any other connected vertex of $M_{i}$;

2 . if $u$ is not an initial node and $\operatorname{true}(u)$ contains an atomic proposition corresponding to a variable which is negated in $\psi$, then delete $u$ from $M_{i}$.

This transformation can be performed in $O(|\psi| \cdot|\mathcal{M}|)$ time and preserves the reachability of the states of $\mathcal{M}^{\mathcal{F}}$ satisfying $\psi$, thanks to Proposition 2

Now, define a supernode $u \in N_{i}$ as recursively expansible if $i \in \operatorname{expand}^{+}(u)$ and a graph $M_{i}$ as recursively expansible if it contains at least a recursively expansible supernode. We define the equivalence relation $\approx$ on the indices of recursively expansible graphs: $i \approx j$ if and only if vertices $u \in N_{i}$ and $v \in N_{j}$ exist such that $i \in \operatorname{expand}^{+}(v)$ and $j \in \operatorname{expand}^{+}(u)$. We want to define a CHSM $\overline{\mathcal{M}}$ $=\left(\bar{M}_{1}, \bar{M}_{2}, \ldots, \bar{M}_{k^{\prime}}\right)$ such that $\overline{\mathcal{M}}$ has a component graph for each equivalence class of the relation $\approx$. Let $f:\{1, \ldots, k\} \longrightarrow\left\{1, \ldots, k^{\prime}\right\}$ be the function that maps each $i$ to $j$ such that $i$ is in the equivalence class corresponding to $\bar{M}_{j}$. 
For a graph $M_{i}$ which is not recursively expansible (i.e., $[i]=\{i\}$ ), we define $\bar{M}_{f(i)}$ as $M_{i}$ except for the mapping expand, since $\operatorname{expand}_{\overline{\mathcal{M}}}(u)=$ $f\left(\operatorname{expand}_{\mathcal{M}}(u)\right)$. For a recursively expansible graph $M_{i}$ we define $\bar{M}_{f(i)}$ as follows. All vertices $u \in N_{j}$ which are not recursively expansible, with $j \approx i$, are vertices of $\bar{M}_{f(i)}$, the edges between them in $M_{i}$ are edges of $\bar{M}_{f(i)}$ as well and $\mathrm{OUT}_{f(i)}=\bigcup_{j, j \approx i} \mathrm{OUT}_{j}$. Moreover, we add a new initial node $i n_{f(i)}$ and insert edges from $i \bar{n}_{f(i)}$ to all vertices $i n_{j}, j \approx i$. For each supernode $u$ of $\bar{M}_{f(i)}$ we define $\operatorname{expand}_{\overline{\mathcal{M}}}(u)=f\left(\operatorname{expand}_{\mathcal{M}}(u)\right)$. Let $S_{\mathcal{M}}(i)$ be the set of all recursively expansible vertices belonging to all graphs $M_{j}$ such that $j \approx i$. We define $\operatorname{true}_{\overline{\mathcal{M}}}\left(\overline{i n}_{f(i)}\right)$ as $\operatorname{true}_{\overline{\mathcal{M}}}\left(i n_{j}\right)$ for an arbitrary $j \approx i$, and for each vertex $u$ of $\bar{M}_{f(i)}, \operatorname{true}_{\mathcal{M}}(u)$ as $\bigcup_{v \in S_{\mathcal{M}}(i)} \operatorname{true}_{\mathcal{M}}(v) \cup \operatorname{true}_{\mathcal{M}}(u)$ (note that no atomic proposition added in this way to the label of $u$ corresponds to a variable which is negated in $\psi$ ).

Now observe that, by the above part 2 of the above simplification, if $X$ is a state of $\mathcal{M}^{F}$ satisfying $\psi$ and $Y$ is a state of $\overline{\mathcal{M}}^{F}$ such that $\operatorname{true} \mathcal{M}(X) \subseteq$ $\operatorname{true}_{\overline{\mathcal{M}}}(Y)$ and $\operatorname{true}_{\overline{\mathcal{M}}}(Y) \backslash \operatorname{true}_{\mathcal{M}}(X)$ does not contain an atomic proposition corresponding to a variable which is negated in $\psi$, then $Y$ satisfies $\psi$ as well. Since the initial simplification also preserves reachability, we have that if a reachable state of $\mathcal{M}^{F}$ fulfilling $\psi$ exists, then a state of $\overline{\mathcal{M}}^{F}$ fulfilling $\psi$ also exists. Since by construction, states of $\overline{\mathcal{M}}^{F}$ corresponds to states of $\mathcal{M}^{F}$, the vice-versa also holds.

As a consequence of Theorem 3 and the arguments for CHSMs and DNF formulas, the following theorem holds.

Theorem 4. The reachability and cycle detection problems on a CRSM $\mathcal{M}$ and a propositional boolean formula $\varphi$ are decidable in $O\left(|\mathcal{M}| \cdot 2^{|\varphi|}\right)$ time.

\section{$5 \quad$ LTL Model Checking}

Here we consider the verification problem of linear-time requirements, expressed by LTL-formulas [Pnu77]. We follow the automata theoretic approach to solving model checking [VW86]: given an LTL formula $\varphi$ and a Kripke structure $M$, it is possible to reduce model checking to the emptiness problem of Büchi automata. To use this approach, we extend the Cartesian product between Kripke structures.

Given a transition graph with states labeled by subsets of atomic propositions and a state $s$, a trace is an infinite sequence $\alpha_{1} \alpha_{2} \ldots \alpha_{i} \ldots$ of labels of states occuring in a path starting from $s$. Moreover, given a CRSM $\mathcal{M}$, we define the language $\mathcal{L}(\mathcal{M})$ as the set of the traces of $\mathcal{M}^{F}$ starting from its initial state. A Büchi automaton $A=\left(Q, q_{1}, \Delta, L, T\right)$ is a Kripke structure $(Q, \Delta, L)$ together with a set of accepting states $T$ and a starting state $q_{1}$. The language $\mathcal{L}(A)$ accepted by $A$ is the set of the traces corresponding to paths visiting infinitely often a state of $T$.

Let $\mathcal{M}=\left(M_{1}, \ldots, M_{k}\right)$ be a CRSM and $A=\left(Q, q_{1}, \Delta, L, T\right)$, for $Q=$ $\left\{q_{1}, \ldots, q_{m}\right\}$, be a Büchi automaton. Let $1 \leq i \leq k, 1 \leq j \leq m$, and $P$ be such 
that $P \subseteq A P$ and $P \cup \operatorname{true}_{\mathcal{M}}\left(i n_{i}\right)=L\left(q_{j}\right)$, we define the graphs $M_{(i, j, P)}$ as follows. Each $M_{(i, j, P)}$ contains vertices $[u, q, j, P]$ such that $(u, q)$ belongs to the standard Cartesian product of $M_{i}$ and $A$, and the labeling of $q$ coincides with the labeling of $u$ augmented with the atomic propositions that $u$ inherits from its ancestors in a given context. The inherited set of atomic propositions is given by $P$. The property $P \cup \operatorname{true}_{\mathcal{M}}\left(i n_{i}\right)=L\left(q_{j}\right)$ assures that we consider only graphs $M_{(i, j, P)}$ whose initial vertex is compatible with the automaton state. Formally, we have:

- The set $N_{(i, j, P)}$ of the vertices of $M_{(i, j, P)}$ contains quadruples $[u, q, j, P]$, where $u \in N_{i}, q \in Q$, and

- either $\operatorname{expand}_{\mathcal{M}}(u)=0$ and $L(q)=\operatorname{true}_{\mathcal{M}}(u) \cup P$

- or $\operatorname{expand}_{\mathcal{M}}(u)=h>0$ and $L(q)=\operatorname{true}_{\mathcal{M}}(u) \cup \operatorname{true}_{\mathcal{M}}\left(i n_{h}\right) \cup P$.

- The initial vertex of $M_{(i, j, P)}$ is $\left[i n_{i}, q_{j}, j, P\right]$ and the output nodes are $[u, q, j, P]$ for $u \in O U T_{i}$ and $q \in Q$;

- $M_{(i, j, P)}$ contains the following edges:

- $\left(\left[u, q^{\prime}, j, P\right],\left[v, q^{\prime \prime}, j, P\right]\right)$, with $\left(q^{\prime}, q^{\prime \prime}\right) \in \Delta$ and $(u, v) \in E_{i}$,

- $\left(\left(\left[u, q_{j^{\prime}}, j, P\right],\left[z, q^{\prime}, j^{\prime}, P \cup \operatorname{true} \mathcal{\mathcal { M }}(u)\right]\right),\left[v, q^{\prime \prime}, j, P\right]\right)$, with $\left(q^{\prime}, q^{\prime \prime}\right) \in \Delta$, $((u, z), v) \in E_{i}$, and $L(q)=\operatorname{true}_{\mathcal{M}}(u) \cup \operatorname{true}_{\mathcal{M}}\left(i_{h}\right) \cup P$ for $\operatorname{expand}_{\mathcal{M}}(u)=h$.

From the above definition we observe that if $u$ is a supernode then the labeling of $q$ has to match also with the labeling of $i n_{\text {expand }}(u)$ since $[u, q, j, P]$ is a supernode of $\mathcal{M}^{\prime}$ and one has to assure the correctness, with respect to the labeling, of its expansion. Note that when only the value of $j$ varies, we have graphs which differ one each other only for the choice of the the initial vertex $\left[i n_{i}, q_{j}, j, P\right]$. Moreover, the edges in $M_{(i, j, P)}$ are given by coupling the transitions $\left(q^{\prime}, q^{\prime \prime}\right)$ of $A$ with both kinds of edges $(u, v)$ and $((u, z), v)$ in $E_{i}$. For $h=\operatorname{expand}_{\mathcal{M}}(u)$, we have edges $\left(\left([u, q, j, P],\left[z, q^{\prime}, h, P \cup \operatorname{true} e_{\mathcal{M}}(u)\right]\right),\left[v, q^{\prime \prime}, j, P\right]\right)$ for every $q \in Q$ such that $L(q)=\operatorname{true}_{\mathcal{M}}(u) \cup \operatorname{true}_{\mathcal{M}}\left(i n_{h}\right) \cup P$. Thus, there might be as many as $|Q|$ edges, for every pair of edges $((u, z), v)$ and $\left(q^{\prime}, q^{\prime \prime}\right)$.

We can now define $\mathcal{M}^{\prime}=\mathcal{M} \otimes A$ as a CRSM constituted by some of the graphs $M_{(i, j, P)}$, and defined inductively as follows:

- $M_{(k, 1, \emptyset)}$ is the graph containing the starting node of $\mathcal{M}^{\prime}$;

- Let $M_{(i, j, P)}$ be a graph of $\mathcal{M}^{\prime}$, and $\left[u, q_{t}, j, P\right]$ be a vertex of $M_{(i, j, P)}$.

- If $\operatorname{expand}_{\mathcal{M}}(u)=0$ then $\operatorname{expand}_{\mathcal{M}^{\prime}}\left(\left[u, q_{t}, j, P\right]\right)=0$;

- If expand $\operatorname{ex}_{\mathcal{M}}(u)=h>0$, and $P^{\prime}=P \cup \operatorname{true} e_{\mathcal{M}}(u)$ then $M_{\left(h, t, P^{\prime}\right)}$ is a graph of $\mathcal{M}^{\prime}$ and $\operatorname{expand}_{\mathcal{M}^{\prime}}\left(\left[u, q_{t}, j, P\right]\right)=\left\langle h, t, P^{\prime}\right\rangle$ where $\left\langle h, t, P^{\prime}\right\rangle$ denotes the index of $M_{\left(h, t, P^{\prime}\right)}$

$-\operatorname{true}_{\mathcal{M}^{\prime}}([u, q, j, P])=\operatorname{true}_{\mathcal{M}}(u)$, for every $[u, q, j, P]$.

Observe that $\mathcal{M}^{\prime}=\mathcal{M} \otimes A$ is a CRSM and if $\mathcal{M}$ is a CHSM, then $\mathcal{M}^{\prime}$ is a CHSM, as well. To determine the size of $\mathcal{M}^{\prime}$, first consider the size of each graph $M_{(i, j, P)}$. The number of the edges is bounded by the product of the number of edges in $M_{i}$ and the number of transitions in $A$ multiplied at most by $m$, since we have at most $|Q|$ edges for any $\left(q^{\prime}, q^{\prime \prime}\right) \in \Delta$ and $((u, z), v) \in E_{i}$. Thus, an upper bound to the size of $M_{(i, j, P)}$ is given by $\left(m \cdot\left|E_{i}\right| \cdot|A|\right)$. The size of $\mathcal{M}^{\prime}$ can be obtained now by counting the number of its component graphs. 
Lemma 4. Given a CRSM $\mathcal{M}, \mathcal{M}^{\prime}=\mathcal{M} \otimes A$ is a CRSM that can be constructed in $O\left(m^{2} \cdot|\mathcal{M}| \cdot|A| \cdot\left|2^{A P}\right|\right)$ time. Moreover, if $\mathcal{M}$ is a $\mathbf{C H S M}$, then $\mathcal{M}^{\prime}$ is a CHSM that can be constructed in $O\left(m^{2} \cdot|\mathcal{M}| \cdot|A|\right)$ time.

Proof First recall that a graph $M_{(i, j, P)}$ of $\mathcal{M}^{\prime}$ has the property that $P \cup$ $\operatorname{true}_{\mathcal{M}}\left(i n_{i}\right)=L\left(q_{j}\right)$. Therefore, $P$ is the union of two disjoint sets $P_{1}$ and $P_{2}$, such that $P_{1}$ is the set of the atomic propositions of $L\left(q_{j}\right)$ that do not belong to $\operatorname{true}_{\mathcal{M}}\left(i n_{i}\right)$, and $P_{2}=P \cap \operatorname{true} e_{\mathcal{M}}\left(i n_{i}\right)$ is a subset of $\operatorname{true} e_{\mathcal{M}}\left(i n_{i}\right)$. Thus, for fixed values of $i$ and $j, P_{1}$ is fixed and the number of different graphs $M_{(i, j, P)}$ is bounded above by the number of different subsets of $\operatorname{true}_{\mathcal{M}}\left(i n_{i}\right)$. Therefore, the size of $\mathcal{M}^{\prime}$ is bounded above by $\sum_{j=1}^{m} \sum_{i=1}^{k}\left(2^{|A P|} \cdot m \cdot\left|M_{i}\right| \cdot|A|\right)$.

Now, let $\mathcal{M}$ be a CHSM. Given a graph $M_{(i, j, P)}$ of $\mathcal{M}^{\prime}, P$ is defined as the set of the propositions that the vertices of $M_{i}$ inherit. Thus, $P \cap \operatorname{true} \mathcal{M}(u)=\emptyset$, for every vertex $u$ of $M_{i}$ and then $P \cap \operatorname{true}_{\mathcal{M}}\left(i n_{i}\right)=\emptyset$. Hence, in this case, $P_{2}$ is empty and then at most one graph $M_{(i, j, P)}$ exists for fixed values of $i$ and $j$. Therefore, the size of $\mathcal{M}^{\prime}$ is bounded above by $\sum_{j=1}^{m} \sum_{i=1}^{k}\left(m \cdot\left|M_{i}\right| \cdot|A|\right)$.

The CRSM $\mathcal{M} \otimes A$ can be used to check for the emptiness of the language given by the intersection of $\mathcal{L}(\mathcal{M})$ and $\mathcal{L}(A)$, as shown in the following lemma.

Lemma 5. There exists an algorithm checking whether $\mathcal{L}(\mathcal{M}) \cap \mathcal{L}(A)=\emptyset$ in time linear in the size of $\mathcal{M}^{\prime}=\mathcal{M} \otimes A$.

Proof First, observe that if we consider as set of final states the vertices $[u, q, h, P]$ such that $q \in T$, the $\mathbf{C R S M} \mathcal{M}^{\prime}$ is a recursive Büchi automaton. Moreover, the set of the traces of $\mathcal{M}^{\prime F}$ is the same as the set of traces of the Cartesian product of $\mathcal{M}^{F}$ and $A$. Thus $\mathcal{L}(\mathcal{M}) \cap \mathcal{L}(A) \neq \emptyset$ if and only $\mathcal{L}\left(\mathcal{M}^{\prime}\right) \neq \emptyset$. From [AEY01, for recursive Büchi automata with a single initial node for each graph, non-emptiness can be checked in linear time.

As a consequence of the above lemmas, we obtain an algorithm to solving the LTL model checking for CRSMs. Following the automata theoretic approach, one can construct a Büchi automaton $A_{\neg \varphi}$ of size $O\left(2^{|\varphi|}\right)$ accepting the set $\mathcal{L}\left(A_{\neg \varphi}\right)$ of the sequences which do not satisfy $\varphi$, and then $\varphi$ is satisfied on all paths of $\mathcal{M}$ if and only if $\mathcal{L}(\mathcal{M}) \cap \mathcal{L}\left(A_{\neg \varphi}\right)$ is empty. From Lemma 4 one can now construct $\mathcal{M} \otimes A_{\neg \varphi}$, whose size is $O\left(m^{2} \cdot|\mathcal{M}| \cdot\left|A_{\neg \varphi}\right| \cdot 2^{|A P|}\right)=O\left(|\mathcal{M}| \cdot 16^{|\varphi|}\right)$ (since $m=\left|A_{\neg \varphi}\right|=O\left(2^{|\varphi|}\right.$ ) and $2^{|A P|} \leq 2^{|\varphi|}$ ). Moreover, this size reduces to $O\left(m^{2} \cdot|\mathcal{M}| \cdot\left|A_{\neg \varphi}\right|\right)=O\left(|\mathcal{M}| \cdot 8^{|\varphi|}\right)$, when $\mathcal{M}$ is a CHSM. Hence, by Lemma 5 we obtain the main result of this section.

Theorem 5. The LTL model checking on a CRSM $\mathcal{M}$ and a formula $\varphi$ can be solved in $O\left(|\mathcal{M}| \cdot 16^{|\varphi|}\right)$ time. Moreover, if $M$ is a CHSM the problem can be solved in $O\left(|\mathcal{M}| \cdot 8^{|\varphi|}\right)$ time.

\section{Discussion}

We have proposed new abstract models for sequential state machines: the context-dependent hierarchical and recursive state machines. On these models we have studied reachability, cycle detection and the more general problem 
of model checking with respect to linear-time specifications. An interesting feature of CHSMs is that they allow very succinct representations of systems, and this comes substantially at no cost if compared to analogous hierarchical models studied in the literature. Moreover, we prove that for some particular formulas we improve the complexity of previous approaches.

Several extensions of the introduced models can be considered.

Our models are sequential. If we add concurrency to CHSMs, the computational complexity of the considered decision problems grows significantly (we recall that reachability in communicating hierarchical state machines is EXPSPACEcomplete [AKY99]). While for CRSMs with concurrency, reachability becomes undecidable since sequential CRSMs are as expressive as pushdown automata AEY01BGR01.

We have only considered models where a single entry node is allowed for each component machine. We can relax this limitation allowing multiple entry points. The semantics of this extension naturally follows from the semantics given for the single entry case. In the hierarchic setting, we can translate a multiple-entry CHSM $\mathcal{M}$ into an equivalent single-entry CHSM $\mathcal{M}^{\prime}$ of size at most cubic in the size of $\mathcal{M}$. In fact, each component machine of $\mathcal{M}$ can be replaced in $\mathcal{M}^{\prime}$ by multiple copies, each copy corresponding to an entry point and having as unique entry point the entry point itself. Expansions are redirected to the proper components in order to match the expansions in $\mathcal{M}$. Thus, supernodes may need to be replaced by multiple copies each pointing to the proper machine in $\mathcal{M}^{\prime}$. If we apply this construction to a multiple-entry CRSM, the obtained single-entry CRSM does not satisfy the property $\operatorname{true}(u) \cap \operatorname{true}(v)=\emptyset$, for $v \in N_{h}, u \notin N_{h}$ and $h \in \operatorname{expand}^{+}(u)$ (see definition of CRSM). This is a consequence of the fact that if a machine of the multiple-entry CRSM can directly or indirectly call itself, then there are two copies of this machine that may call each other recursively. We recall that the above property is sufficient to ensure that Condition 1 holds for conjunctions of literals, and thus is crucial to obtain the results given in Section 4 However, it is possible to prove that Theorem 3 also holds for multiple-entry CRSMs. We leave the details of this proof to the full paper.

For modeling purposes it is useful to have variables over a finite domain that can be passed from a component to another. We can extend our models to handle input, output and local variables. Consider a component machine $M$ with $h_{e}$ entry nodes, $h_{x}$ exit nodes, and $h_{t}$ internal vertices. If $M$ is equipped also with $k_{i}$ input boolean variables, $k_{o}$ output boolean variables, and $k_{l}$ local boolean variables, we can model by a machine having $2^{k_{i}} \cdot h_{e}$ entry nodes, $2^{k_{o}} \cdot h_{x}$ exit nodes, and $2^{k_{i}+k_{l}+k_{o}} \cdot h_{t}$ internal vertices.

\section{References}

[AEY01] R. Alur, K. Etessami, and M. Yannakakis. Analysis of recursive state machines. In Proc. of the 13th International Conference on Computer Aided Verification, CAV'01, LNCS 2102, pages 207-220. Springer, 2001. 
[AG00] R. Alur and R. Grosu. Modular refinement of hierarchic reactive machines. In Proc. of the 27th Annual ACM Symposium on Principles of Programming Languages, pages 390-402, 2000.

[AGM00] R. Alur, R. Grosu, and M. McDougall. Efficient reachability analysis of hierarchical reactive machines. In Computer Aided Verification, 12th International Conference, LNCS 1855, pages 280-295. Springer, 2000.

[AKY99] R. Alur, S. Kannan, and M. Yannakakis. Communicating hierarchical state machines. In Proc. of the 26-th International Colloquium on Automata, Languages and Programming, ICALP'99, LNCS 1644, pages 169178. Springer-Verlag, 1999.

[AY01] R. Alur and M. Yannakakis. Model checking of hierarchical state machines. ACM Transactions on Programming Languages and Systems (TOPLAS), 23(3):273-303, 2001.

[BGR01] M. Benedikt, P. Godefroid, and T. W. Reps. Model checking of unrestricted hierarchical state machines. In Proc. of the 28th International Colloquium Automata, Languages and Programming, ICALP'01, LNCS 2076, pages 652-666. Springer, 2001.

[BJR97] G. Booch, I. Jacobson, and J. Rumbaugh. Unified Modeling Language User Guide. Addison Wesley, 1997.

$\left[\mathrm{BLA}^{+} 99\right]$ G. Behrmann, K.G. Larsen, H.R. Andersen, H. Hulgaard, and J. LindNielsen. Verification of hierarchical state/event systems using reusability and compositonality. In Proc. of the Tools and Algorithms for the Construction and Analysis of Systems, TACAS'99, LNCS 1579, pages 163-177. Springer, 1999.

[CE81] E.M. Clarke and E.A. Emerson. Design and synthesis of synchronization skeletons using branching time temporal logic. In Proc. of Workshop on Logic of Programs, LNCS 131, pages 52-71. Springer-Verlag, 1981.

[CK96] E.M. Clarke and R.P. Kurshan. Computer-aided verification. IEEE Spectrum, 33(6):61-67, 1996.

[Pnu77] A. Pnueli. The temporal logic of programs. In Proc. of the 18th IEEE Symposium on Foundations of Computer Science, pages 46-77, 1977.

$\left[\mathrm{RBP}^{+} 91\right]$ J. Rumabaugh, M. Blaha, W. Premerlani, F. Eddy, and W. Lorensen. Object-oriented Modeling and Design. Prentice-Hall, 1991.

[SGW94] B. Selic, G. Gullekson, and P.T. Ward. Real-time object oriented modeling and design. J. Wiley, 1994.

[VW86] M.Y. Vardi and P. Wolper. Automata-theoretic techniques for modal logics of programs. Journal of Computer and System Sciences, 32:182-211, 1986. 